\title{
THE GEOMETRY AND ARITHMETIC OF TRANSLATION SURFACES WITH APPLICATIONS TO POLYGONAL BILLIARDS
}

\author{
Eugene Gutkin and Chris Judge
}

\begin{abstract}
A translation manifold is a manifold whose transition transformations are translations. There is an important connection between the geometry and arithmetic of translation surfaces and dynamics of polygonal billiards. There are also remarkable relations with automorphic forms. In this note we announce results which further develop these connections.
\end{abstract}

\section{Introduction}

Let $P \subset R^{2}$ be an arbitrary polygon. It is rational if the angles between the sides of $P$ are of the form $\pi m / n$. It has been known since the work of Katok and Zemlyakov [KZ75] that the billiard dynamics in $P$ is essentially equivalent to the geodesic flow on a closed oriented surface, $S=S(P)$, endowed with a flat Riemannian metric, with special singularities. The geodesic flow decomposes into a one-parameter family of directional flows on $S$. These flows are closely related to interval exchange transformations, on one hand, and to holomorphic quadratic differentials on Riemann surfaces, on the other. These relationships have proven fruitful. For example, they were used to obtain strong results on ergodicity for interval exchanges [Mas82, Vch82], directional billiards in rational polygons [KMS], and full billiards in irrational polygons [Kat86, KMS]. We refer the reader to the surveys [Gut86, Gut95] for more information on the subject, and turn now to the geometric aspects of the connection between polygons and surfaces.

The genus of $S=S(P)$ is determined by the angles of $P$ [Gut84]. In particular, $g(S) \geq 1$, and the equality holds only for very special $P$. But the other natural structures on $S(P)$ depend on $P$ in a subtle way. In particular, $S$ has a special $(G, X)$-structure [Thurst78], the translation structure, which makes it a translation surface (Definition 1$)$.

Received February 19, 1996.

1991 Mathematics Subject Clasification 30F60; Secondary 30F30, 58F17, 11F72.

The work of the first author is partially supported by NSF grants DMS-9013220 and DMS-9400295.

The work of the second author is supported by an NSF postdoctoral fellowship. 
Let $S$ be any translation surface. The group of affine diffeomorphisms of $S$ has a natural homomorphism into $S L(2, R)$. The image, $\Gamma=\Gamma_{S}$, is a discrete subgroup of $S L(2, R)$ [Vch89], and we call it the Veech group of the translation surface $S .{ }^{1}$ If $S=S(P)$, we set $\Gamma_{P}=\Gamma_{S(P)}$, and call it the Veech group of the polygon $P$. A translation surface $S$ (rational polygon $P$ ) is a Veech surface (Veech polygon) if the group $\Gamma_{S}\left(\Gamma_{P}\right)$ is cofinite with respect to the Haar measure. (Veech groups are never cocompact [Vch89].)

Let $S$ be a translation surface. Periodic geodesics on $S$ naturally form Euclidean cylinders, imbedded in $S$. Let $\ell_{1} \leq \ell_{2} \leq \cdots$ be the lengths of distinct maximal periodic cylinders. Define the zeta function

$$
\zeta_{S}(t)=\sum_{i=1}^{\infty}\left(\ell_{i}\right)^{-2 t}
$$

and let $b_{S}(x)$ be the number of cylinders of length at most $x$. If $S$ is a Veech surface, and $\Gamma=\Gamma_{S}$, then there is a remarkable expression for $\zeta_{S}$ in terms of a combination of Eisenstein series associated to the cusps of $\Gamma$ [Vch89]. Using classical facts about the Eisenstein series, Veech proved a prime geodesic theorem, that is $b_{S}(x) \sim c(S) x^{2}$, where $c(S)>0$ involves the hyperbolic area of $H^{2} / \Gamma$ [Vch89].

Let $\Gamma \subset S L(2, R)$ be an arbitrary cofinite (not cocompact) lattice, and let $\Delta_{\Gamma}$ be the Laplace-Beltrami operator on the hyperbolic surface $H^{2} / \Gamma$. The spectrum of $\Delta_{\Gamma}$ is divided into the continuous and the discrete parts, and the Eisenstein series represent the continuous part [Trs, Vnk]. P. Sarnak has conjectured that the asymptotic density of the continuous spectrum is dominated by that of the discrete spectrum if and only if $\Gamma$ is arithmetic [Srk86].

Let now $\Gamma$ be the Veech group of a Veech surface $S$. Sarnak suggested to one of us (C. J.) that the relation of $\zeta_{S}$ to the Eisenstein series of $\Gamma$ might be used to obtain information about the asymptotic density of the discrete spectrum of $H^{2} / \Gamma$. In Theorem 4 and Corollary 2 below, we provide a geometric criterion for the arithmeticity of Veech groups, and thus obtain a geometric reformulation of Sarnak's conjecture in this context (see $\S 5$, Conjecture 1).

If $S=S(P)$, then closed geodesics in $S$ correspond to periodic billiard orbits in $P$, and we denote by $b_{P}(x)$ the counting function of these orbits. By results of Masur [Mas88, Mas90], for any rational polygon, $b_{P}(x)$ has quadratic upper and lower bounds. For general irrational polygons, the function $b_{P}(x)$ grows subexponentially [Kat87, GH95, GH96], and no

\footnotetext{
${ }^{1}$ The Veech group of a translation structure is the direct analog of a subgroup of $P S L(2, R)$ that Veech associates with an " $F$-structure" [Vch89, Vch93]. It is also closely related to the Teichmuller disc stabilizer group, see, e. g., [Kra81, Thurst88, Krb78].
} 
lower bounds on $b_{P}(x)$ are known. Certain classes of irrational polygons (including arbitrary right triangles) have infinitely many periodic orbits [GT95].

The facts above suggest a number of questions. Let $\Gamma \subset S L(2, R)$ be the Veech group of a rational polygon $P$ or a translation surface $S$. Is $\Gamma$ nontrivial? When is $\Gamma$ a lattice? A nonelementary group? Which discrete subgroups of $S L(2, R)$ occur as Veech groups? More generally, what are the relations between $\Gamma$ and the geometric/dynamic properties of $P$ ?

In this note we announce our results that shed some light on these questions. Details will appear in [GJ96] and elsewhere. It is perhaps not surprising that Veech surfaces are scarce among translation surfaces of a fixed genus, $g>1$ (Theorem 1). However, if we consider translation surfaces of all genera, then their Veech groups behave naturally under coverings (Theorems 2 and 3 ). This observation allows us to exhibit whole new classes of Veech surfaces (Corollary 1, Theorem 5). In particular, our results yield many new examples of Veech polygons (Proposition 3). Until now the only nontrivial examples were the isoceles triangles with base angles $\pi / n$ [Vch89], the regular polygons [Vch92], and the $(\pi / n, \pi / 2 n, \pi(2 n-3) / 2 n)$ triangles [Wrd96]. The Veech groups in question are not arithmetic, if $n \neq 3,4,6$. Theorem 4 characterizes in geometric terms the Veech surfaces with arithmetic Veech groups; they are affine coverings of flat tori with at most one branch point. For Veech surfaces of genus greater than one, Corollary 2 reformulates this criterion in terms of branched coverings of elliptic curves.

\section{Translation surfaces}

In this section we introduce the basic definitions and facts to be used later on. (Compare with [Vch89], §2.) Let $g \geq 1$, and let $S=S_{g}$ be the (connected, closed, oriented, differentiable) surface of genus $g$. Let $\Sigma \subset S$ be a finite set, and let $f_{i}: U_{i} \rightarrow R^{2}, i \in I$, be a coordinate atlas on $S \backslash \Sigma$. It is a translation atlas if the transitions $f_{i} f_{j}^{-1}$ are translations, $f_{i} f_{j}^{-1}=t_{i j} \in R^{2}$. Two translation atlases are equivalent if their union is a translation atlas. A translation structure on $S \backslash \Sigma$ is an equivalence class of translation atlases (representatives of the translation structure). Let $u$ be a translation structure on $S \backslash \Sigma$, let $\sigma \in \Sigma$, and let $V \subset S$ be a contractible neighborhood, such that $V \cap \Sigma=\{\sigma\}$. A representative of $u$ defines a mapping, $f: V \backslash\{\sigma\} \rightarrow R^{2}$. The property that $f$ extend, by continuity, to $V$ does not depend on the choices involved; it is a property of the translation structure. If it holds for all $\sigma \in \Sigma$, we say that $u$ is conically closable, and that $\sigma \in \Sigma$ are the cone points. 
Definition 1. A translation surface of genus $g$ is a translation structure on $S_{g} \backslash \Sigma$. In what follows we assume that $|\Sigma|<\infty$, and the translation structure is conically closable.

Some of the cone points of a translation surface may be 'spurious singularities', in the sense that the cone angle (see below) is $2 \pi$. There are two points of view on this problem. According to one, we denote by $\mathcal{U}_{g}$ the set of translation surfaces in genus $g$, and consider the cone set, $\Sigma_{u}$, to be intrinsically determined by $u \in \mathcal{U}_{g}$. Then $\sigma \in \Sigma_{u}$ are genuine cone points, with cone angles greater than $2 \pi$. From the other viewpoint, the cone set $\Sigma \subset S$ is a part of the data for a translation surface. We use both viewpoints here. When using the former, we denote our translation surface by $S_{u}$. The latter requires notation $(S, \Sigma)$.

Let $S_{u}$ be a translation surface, $u \in \mathcal{U}_{g}$. We suppress $g$ and $u$ from notation if confusion is unlikely. Via a translation atlas, we pull back the Riemannian metric $d s^{2}=d x^{2}+d y^{2}$ and the form $d z=d x+i d y$ from $R^{2}$ to $S \backslash \Sigma$. Then $S$ is the metric completion of $S \backslash \Sigma$ with respect to this metric. The pull-back metric is flat on $S \backslash \Sigma$, and the points in $\Sigma$ are conical singularities [Jdg95b]. The cone angle at $\sigma \in \Sigma$ is $2 \pi n_{\sigma}, n_{\sigma}>1$, an integer.

The pull-back of $d z$ uniquely extends to $S$, as a holomorphic one-form, $\alpha_{u}$, with respect to a uniquely defined conformal structure on $S$. The cone points $\sigma \in \Sigma_{u}$ are the zeros of $\alpha_{u}$, and $n_{\sigma}-1>0$ is the order of zero.

Proposition 1. Let $g \geq 1$. The construction outlined above yields a oneto-one correspondence between the set of translation surfaces and the set of pairs (complex structure, holomorphic one-form), in genus $g$.

As a byproduct of Proposition 1, we obtain the formula

$$
g=1+\frac{1}{2} \sum_{\sigma \in \Sigma} n_{\sigma}-\frac{1}{2}|\Sigma|
$$

expressing the genus in terms of cone angles of a translation structure. Note that it suffices to set $\Sigma=\Sigma_{u}$ in eq. (2), but it is valid for any $\Sigma$.

Let Diff $\left(D i f f_{0}\right)$ be the group of orientation preserving (homotopic to the identity) diffeomorphisms of $S$, and let Map $=$ Diff $/$ Dif $f_{0}$ be the mapping class group. Let $G L_{+}(2, R)$ be the usual linear group of $R^{2}$. The natural actions of Diff and $G L_{+}(2, R)$ on $\mathcal{U}$ commute, defining an action of $G L_{+}(2, R) \times$ Diff.

Definition 2. A nontrivial complex one-form $\alpha$ on $S$ is called geometric if $\alpha$ is holomorphic with respect to a (necessarily unique) complex structure on $S$. 
The group $G L_{+}(2, R) \times$ Diff naturally acts on the set, $\mathcal{A}$, of geometric forms.

Proposition 2. The one-to-one correspondence of Proposition 1 yields an isomorphism between $\mathcal{U}$ and $\mathcal{A}$, equivariant with respect to the actions of $G L_{+}(2, R) \times$ Diff.

It is convenient to have Diff act on the right of both $\mathcal{U}$ and $\mathcal{A}$. For $\phi \in \operatorname{Diff}$, we will use notation $u \mapsto u \phi$, and $\alpha \mapsto \alpha \phi^{*}$ for the two actions. We also denote by $\phi^{*}$ the induced action of Map on the cohomology of $S$.

Definition 3. Two translation structures, $u, v \in \mathcal{U}$ are affinely equivalent, if there exists $g \in G L_{+}(2, R)$, such that $v=g u$.

Definition 4. (Compare with [Vch89]) Let $u \in \mathcal{U}$. The group, Af $f_{u} \subset$ Diff , of affine diffeomorphisms consists of $\phi$, such that $u \phi=g u$, for some $g \in G L_{+}(2, R)$.

If $\phi \in A f f_{u}$, then the element $g=d_{u}(\phi)$ is uniquely defined, and $d_{u}$ is a homomorphism of $A f f_{u}$ into $S L(2, R)$. The range, $\Gamma_{u}$, of $d_{u}$ is the Veech group of the translation structure $u$. It is immediate that the conjugacy class of $\Gamma_{u}$ depends only on the affine equivalence class of $u$; the stabilizer of $u$ in $G L_{+}(2, R) \times D i f f$ consists of $(g, \phi)$, such that $\phi \in A f f_{u}$, and $g=d_{u}(\phi)$.

\section{Main results}

In view of the results of Veech [Vch89], it is important to have a grasp on Veech groups of translation surfaces. We note that closely related questions have been formulated before: [Kra81], [Thurst88], and [Krb78], problem $2.7 .^{2}$ If $\Gamma_{u}$ is a lattice in $S L(2, R)$, we say that $S_{u}$ is a Veech surface. If $\Gamma_{u}$ belongs to the centre of $S L(2, R)$, we say that the Veech group of $S_{u}$ is trivial. The group $\operatorname{Diff} f_{0}$ acts freely on $\mathcal{U}$. We denote by $\langle\mathcal{U}\rangle$ the quotient space, and let $u \mapsto\langle u>$ be the projection. By Proposition 1, $\langle\mathcal{U}\rangle_{g}$ is fibered over the Teichmuller space, $\mathcal{T}_{g}$, in genus $g$ [Gard87, Nag]. The fiber at a point of $\mathcal{T}_{g}$ consists of nontrivial holomorphic one-forms on the corresponding Riemann surface. Let $g>1$. Then, by a result of Bers [Brs60, Nag], $\langle\mathcal{U}\rangle_{g}$ is a complex analytic manifold of $4 g-3$ dimensions.

The Veech group of a translation structure, $u \in \mathcal{U}$, is determined by the class, $\langle u\rangle \in\langle\mathcal{U}\rangle$, and we will often use notation $\Gamma_{<u\rangle}$.

Theorem 1. Let $g\rangle$. The set of $\langle u\rangle \in\langle\mathcal{U}\rangle$, such that $\Gamma_{<u\rangle}$ is nontrivial, is a union of a countable (at most) number of closed proper submanifolds.

\footnotetext{
${ }^{2}$ We thank G. Mess, who pointed out the reference [Krb78] to us.
} 
Our further results have to do with the behavior of Veech groups under affine mappings (affine coverings) of translation surfaces. From now on we vary the genus, and switch to the viewpoint that requires notation $(S, \Sigma)$ for a translation surface. We denote by $A f f_{(S, \Sigma)}$ the group of affine diffeomorphisms that preserve $\Sigma$. The Veech group, $\Gamma_{(S, \Sigma)} \subset S L(2, R)$, of a translation surface is defined the same way as before (see Definition 4).

Definition 5. Let $\left(S_{1}, \Sigma_{1}\right)$ and $\left(S_{2}, \Sigma_{2}\right)$ be translation surfaces. Let $\phi$ : $S_{1} \rightarrow S_{2}$ be an orientation preserving differentiable submersion, such that $\Sigma_{2} \subset \phi\left(\Sigma_{1}\right)$, and $\phi$ is compatible with the translation structures on $S_{1} \backslash \Sigma_{1}, S_{2} \backslash \Sigma_{2}$. Then $\phi:\left(S_{1}, \Sigma_{1}\right) \rightarrow\left(S_{2}, \Sigma_{2}\right)$ is an affine mapping of translation surfaces.

The natural parallelization of a translation surface identifies the differential of an affine mapping, $\phi$, of translation surfaces with a constant matrix, $d \phi \in G L_{+}(2, R)$. It behaves naturally under compositions of affine mappings. Topologically, $\phi: S_{1} \rightarrow S_{2}$ is a covering, which is, in general, branched. The branch locus is contained in $\phi\left(\Sigma_{1}\right)$. An affine mapping is an affine covering if $\phi\left(\Sigma_{1}\right)=\Sigma_{2}$, and $\phi: S \backslash \Sigma_{1} \rightarrow S \backslash \Sigma_{2}$ is a (unbranched) topological covering.

Recall that two groups, $\Gamma_{1}, \Gamma_{2} \subset S L(2, R)$ are commensurable, if there exists $g \in S L(2, R)$, such that the group $\Gamma_{1} \cap g \Gamma_{2} g^{-1}$ has finite index in both $\Gamma_{1}$ and $g \Gamma_{2} g^{-1}$. If $g=1$, then $\Gamma_{1}, \Gamma_{2}$ are commensurate. Recall that a noncocompact discrete subgroup, $\Gamma \subset S L(2, R)$, is arithmetic, if it is commensurable with $S L(2, Z)$.

Theorem 2. Let $\phi:\left(S_{1}, \Sigma_{1}\right) \rightarrow\left(S_{2}, \Sigma_{2}\right)$ be an affine covering. Then the Veech groups $\Gamma_{\left(S_{1}, \Sigma_{1}\right)}, \Gamma_{\left(S_{2}, \Sigma_{2}\right)}$ are commensurable.

The case when $\Sigma_{1}, \Sigma_{2}$ are minimal cone sets is especially important. To formulate the corresponding result, we switch back to the former point of view.

Theorem 3. Let $u \in \mathcal{U}_{g}, v \in \mathcal{U}_{h}$, and let $R_{u}, S_{v}$ be the corresponding translation surfaces. If $\phi: R_{u} \rightarrow S_{v}$ is an affine covering, then the Veech groups $\Gamma_{<u>}, \Gamma_{<v>}$ are commensurable.

Corollary 1. Let $S_{1}$ and $S_{2}$ be translation surfaces, and let $\Gamma_{1}, \Gamma_{2}$ be their Veech groups. Suppose there exists an affine covering, $\phi: S_{1} \rightarrow S_{2}$. Then:

1. Either they are both Veech surfaces or neither one is a Veech surface;

2. The two Veech groups are both finite or both infinite;

3. One of the Veech groups is elementary if and only if the other one is;

4. One of the Veech groups contains a pair of noncommuting parabolic elements if and only if the other one does;

5. One of the Veech groups is arithmetic if and only if the other one is. 
A flat torus, $T$, is the simplest example of a translation surface. It is convenient to designate $0 \in T$ as a cone point. The Veech group of $(T,\{0\})$ is $S L(2, Z)$. If a translation surface $(S, \Sigma)$ affinely covers $(T,\{0\})$, then, by Theorem 2 , the group $\Gamma_{(S, \Sigma)}$ is arithmetic. The converse also holds.

Theorem 4. Let $(S, \Sigma)$ be a translation surface. Then the Veech group $\Gamma_{(S, \Sigma)}$ is arithmetic if and only if $(S, \Sigma)$ affinely covers a flat torus, with at most one branch pont.

Let $g \geq 1$. Recall that a translation surface in genus $g$, determines a Riemann surface, i. e., a point in the moduli space, $\mathcal{M}_{g}$.

Corollary 2. Let $S$ be a translation surface of genus $g>1$, and let $\Gamma$ be its Veech group. Then $\Gamma$ is arithmetic if and only if $S$, as a Riemann surface, is a branched covering, with exactly one branch point, of an elliptic curve, and the flat structure on $S$ is the pull-back of the canonical flat structure of the elliptic curve.

\section{Examples and applications}

Let $P$ be a connected rational polygon. Orthogonal reflections about the sides of $P$, viewed as elements of $O(2)$, generate a finite dihedral group, $D_{N}$, where $N$ is calculated from the angles of $P$. The group $D_{N}$ acts by isometries on the associated translation surface, $S(P)$, and $P=S(P) / D_{N}$ [Gut84]. Under the projection, $\phi: S(P) \rightarrow P$, the billiard trajectories in $P$ pull back to the geodesics in $S(P)$.

The same construction can be applied to generalized rational polygons. A generalized polygon is a polygonal region located in a branched cover of $R^{2}$. As before, with a generalized polygon we associate a subgroup of $O(2)$. This group is finite if and only if $P$ is rational, in which case it is a dihedral group, $D_{N}$. The translation surface, $S(P)$, and the projection, $\phi: S(P) \rightarrow P=S(P) / D_{N}$, are defined as before.

The rationale for introducing generalized polygons is provided by the concept of unfolding the billiard table. Let $P=P_{1}$ be an ordinary polygon, let $a$ be a side of $P_{1}$, and let $s: R^{2} \rightarrow R^{2}$ be the orthogonal reflection about the line containing $a$. Then the union, $P_{2}=P_{1} \cup s\left(P_{1}\right)$, may not be disjoint. Even if it is, continuing the unfolding, we will eventually arrive at a situation of this type. In this case we think of the obtained region, $P_{n}$, as a generalized polygon.

For the sake of brevity we suppress the adjective 'generalized' in what follows. Let $P$ be any polygon. We denote by $\mathcal{T}(P)$ the set of polygons obtained from $P$ by unfoldings. We say that the polygons $Q \in \mathcal{T}(P)$ are tiled by $P$. 
Definition 6. Two polygons, $Q_{1}, Q_{2}$, are close relatives if they can be tiled by the same polygon, $P$.

The relation above is reflexive, but not necessarily transitive. We use the name relatives for the equivalence relation generated by it. Thus $P, Q$ are relatives $(P \sim Q)$, if there exists a chain, $P_{1}, \ldots, P_{n}$, such that $P=$ $P_{1}, Q=P_{n}$, and $P_{i}, P_{i+1}$ are close relatives. This equivalence relation was used in [GT95] to study periodic billiard orbits in irrational polygons.

Theorem 5. Let $P, Q$ be rational polygons. If $P$ and $Q$ are relatives, then the Veech groups $\Gamma_{P}, \Gamma_{Q}$ are commensurate.

Theorem 5 immediately implies an analog of Corollary 1 for polygons. We leave the formulation to the reader. Note that the analog of assertion 5 is slightly stronger: One of the Veech groups is commensurate with $g S L(2, Z) g^{-1}$ if and only if the other one is.

Combining Theorem 5 with the results of Veech and Ward [Vch89, Wrd96], we obtain many new examples of Veech polygons.

\section{Proposition 3.}

1. For $n>2$ denote by $V_{n}$ the right triangle with angle $\pi / n$. Set $\mathcal{V}_{n}=$ $\mathcal{T}\left(V_{n}\right)$. Then any $P \in \mathcal{V}_{n}$ is a Veech polygon. If $n \neq 4$, then its Veech group is commensurate with the Schwarz $(2, n, \infty)$ triangle group [Brd83]. 2. Let $W_{n}$ be the $(\pi / n, \pi / 2 n, \pi(2 n-3) / 2 n)$ - triangle $(n \geq 3)$. Set $\mathcal{W}_{n}=\mathcal{T}\left(W_{n}\right)$. Then any $P \in \mathcal{W}_{n}$ is a Veech polygon. The Veech group, $\Gamma_{P}$, is commensurate with the Schwarz $(3, n, \infty)$ triangle group.

3. Let $\mathcal{S}$ be the set of polygons that are tiled by the unit square. Then any polygon, $P \in \mathcal{S}$, is a Veech polygon. Its Veech group is commensurate with $S L(2, Z)$.

Note that $\mathcal{V}_{n}$ contains the regular $n$-gon. Thus, assertion 1 of Proposition 3 recovers from a new perspective some of the results of [Vch92].

In another set of examples, $R_{n} \in \mathcal{V}_{n}$, are the rhombi with angles $2 \pi / n, n \geq 3$. Let $n$ be odd. Let $O$ be a vertex of $R_{n}$ with the angle $2 \pi / n$. Unfolding $n$ times $R_{n}$ about $O$, we obtain a star-shaped polygon, $S_{n} \in \mathcal{V}_{n}$, whose angle at $O$ is $2 \pi$. However, $O$ is not an interior point of $S_{n}$, it is a vertex. The angle between the two sides, containing $O$, is equal to zero, thus the sides form a slit [Gut86]. If we unfold the polygon $R_{n}$ about $O 2 n$ times, we obtain a generalized polygon, $T_{n} \in \mathcal{V}_{n}$. It takes a 2-sheeted covering of $R^{2}$, branched at $O$, to imbed $T_{n}$ without self-intersections. Now $O$ is an interior point of $T_{n}$, but it is a cone point, with the cone angle $4 \pi$.

The polygons in part 3 of Proposition 3 were considered in [Gut84] from a different viewpoint, as polygons drawn on a lattice in $R^{2}$. The results 
of [Gut84] on billiards in these polygons now follow from assertion 3 of Proposition 3, and the special properties of geodesics on Veech surfaces [Vch89]. The most important of these properties are:

1. A direction is either completely periodic or completely aperiodic;

2. A prime geodesic theorem holds;

3 . The $\zeta$-function continues meromorphically to the entire complex plane. These properties also hold for billiard orbits in Veech polygons.

However, the properties 1, 2, and 3 do not characterize Veech surfaces (Veech polygons). We have examples of translation surfaces (rational polygons) that satisfy the properties above, but are not Veech surfaces (polygons). These surfaces are constructed as branched coverings of flat tori. ${ }^{3}$

\section{Sketches of proofs and further comments}

First, we outline the ideas behind Theorem 1 . The genus, $g \geq 1$, is fixed, and suppressed from notation, as much as possible. The forms, $\alpha \in \mathcal{A}$, are closed, and let $\alpha \mapsto[\alpha]$ be the natural map from the space of geometric forms to the cohomology, $H^{1}(S, C)$. We denote by $\{\cdot, \cdot\}$ the standard symplectic form on $H^{1}(S, C)$, and let $h \mapsto \bar{h}$ be the conjugation about $H^{1}(S, R)$. The group $G L_{+}(2, R) \times$ Map naturally acts on $H^{1}(S, C)$. The mapping [ ] : $\mathcal{A} \rightarrow H^{1}(S, C)$ is equivariant with respect to the action above, and the action of $G L_{+}(2, R) \times D i f f$ on $\mathcal{A}$. The image, $[\mathcal{A}] \subset H^{1}(S, C)$, is contained in the cone $H_{+}^{1}(S, C)=\{w:\{w, \bar{w}\}>0\}$. The action of $M a p$ on $H^{1}(S, C) \simeq C^{2 g}$ yields a homomorphism of Map onto $\operatorname{Sp}(g, Z)$ [Brm75].

Propositions 1-2, combined with these remarks, yield that the map $c: \mathcal{U} \rightarrow H_{+}^{1}(S, C)$ is equivariant with respect to the actions of $G L_{+}(2, R) \times$ Diff on $\mathcal{U}$, and $G L_{+}(2, R) \times S p(g, Z)$ on $H_{+}^{1}(S, C)$. Let $u \in \mathcal{U}$, and set $w=c(u) \in H_{+}^{1}(S, C)$. Let $L: \operatorname{Diff} \rightarrow S p(g, Z)$ be the composition of homomorphisms Diff $\rightarrow$ Map, and Map $\rightarrow S p(g, Z)$. Then Aff $f_{u}$ is contained in the set of $\phi \in D i f f$, such that $L(\phi)$ stabilizes the real symplectic 2-plane, $[w] \subset H^{1}(S, R)$, spanned by the vectors $\Re w, \Im w$. The action of the stabilizer of $[w]$ in $S p(g, Z)$, restricted to $[w]$, is realized by a matrix group, $I_{w} \subset S L(2, R)$. By the preceding remarks, $\Gamma_{u} \subset I_{w}$. The assertion now follows from the crucial fact that the mapping $\langle c\rangle:\langle\mathcal{U}\rangle$ $\rightarrow H_{+}^{1}(S, C)$ is open.

Now we outline the proofs of Theorems 2 and 3. Let $p:\left(S_{1}, \Sigma_{1}\right) \rightarrow$ $\left(S_{2}, \Sigma_{2}\right)$ be an affine covering. Any affine diffeomorphism, $\phi:\left(S_{2}, \Sigma_{2}\right) \rightarrow$ $\left(S_{2}, \Sigma_{2}\right)$, if it lifts, yields an affine diffeomorphism, $\tilde{\phi}$, of $\left(S_{1}, \Sigma_{1}\right)$. The finiteness of the index of the subgroup of "liftable" affine diffeomorphisms now follows from topological considerations. The finiteness of the index of

\footnotetext{
${ }^{3}$ We thank N. Haydn who pointed out the existence of such coverings to us.
} 
the subgroup of affine diffeomorphisms of $S_{1}$ that descend to $S_{2}$ does not come from topology. In fact, this assertion has no topological counterpart. In our proof, we introduce a special equivalence relation on the set of affine coverings, $p: R \rightarrow S$, where $R$ is fixed, and $S$ varies. The group of affine diffeomorphisms of $R$ naturally acts on the set of equivalence classes. In each affine equivalence class, there is a Riemannian representative, i.e., a covering, $r: R \rightarrow S$, compatible with the flat metrics on translation surfaces (see $\S 2$ ). The assertion now follows from the crucial fact that if $\Sigma_{R} \neq \emptyset$, then there is a finite number of distinct Riemannian coverings, $r_{i}: R \rightarrow S_{i}$.

Theorem 2 implies one half of Theorem 4: If a surface covers a flat torus, with at most one branch point, then its Veech group is arithmetic. To prove the other half of Theorem 4, we use the decompositions of a Veech surface into maximal periodic cylinders. A key observation is that if a Veech group is arithmetic, then the length and the width of any such cylinder are commensurate. This follows from an elementary geometric lemma: If the sides of a triangle have rational slopes, then any side-length is rationally proportional to the opposite height of the triangle.

Next we apply Theorem 4 to reformulate Sarnak's conjecture on 'spectral arithmeticity' [Srk86]. Let $\Gamma \subset S L(2, R)$ be an arbitrary cofinite lattice. Let $M_{\Gamma}(x)$ be the combined number of poles in the disc of radius $x$ of all Eisenstein series of $\Gamma$. The Weyl-Selberg law [Slb54] implies:

$$
\limsup _{x \rightarrow \infty} \frac{M_{\Gamma}(x)}{x^{2}} \leq \frac{\operatorname{Area}\left(H^{2} / \Gamma\right)}{4 \pi} .
$$

A. Selberg conjectured that $M_{\Gamma}(x)=o\left(x^{2}\right)$, i. e., the left hand side of eq. (3) is always zero [Slb54]. Although his conjecture remains unrefuted, there is ample indirect evidence indicating that it is false (see, e. g., [Jdg95a]). In fact, Sarnak has made an antithetical conjecture: $M_{\Gamma}(x)=$ $o\left(x^{2}\right)$ if and only if $\Gamma$ is arithmetic [Srk86].

Let $\Gamma$ be the Veech group of a Veech surface, $S$, and let $\zeta_{S}$ be the zeta function given by equation (1). Using Veech's expression of $\zeta_{S}$ via the Eisenstein series of $\Gamma$ [V ch89], we find that $M_{\Gamma}(x)$ is the number of poles of $\zeta_{S}$ in the disc of radius $x$. Combining this with Corollary 2 of Theorem 4, we reformulate Sarnak's conjecture in purely geometric terms:

Conjecture. Let $\Gamma \subset S L(2, R)$ be the Veech group of a Veech surface, $S$, of genus greater than one. Let $M_{S}(x)$ be the number of poles of the zeta function of $S$ in the disc of radius $x$. Then $M_{S}(x)=o\left(x^{2}\right)$ if and only if $S$, as a Riemann surface, is a branched covering of an elliptic curve, with one branch point, and the flat structure of $S$ is the pull-back of the canonical flat structure of the elliptic curve. 
We point out that the Veech groups of Proposition 3, parts 1 and 2, are nonarithmetic, with the exceptions of $n=3,4,6$ in part 1 , and $n=3,4$ in part 2 [Tak77]. Thus, a refutation of Selberg's conjecture might result from an understanding of the zeta function for these polygons.

\section{Added in proof}

1. After this paper has been accepted for publication, the work [Vrb96], by Y. Vorobets, appeared. The results announced in [Vrb96] overlap with our Theorem 3. The proofs are not given, but, judging by the presentation of material in [Vrb96], they differ considerably from ours. We have presented some of our results to the A.M.S. at the special seesion on Flat Surfaces and Billiard Dynamics at the $905^{\text {th }}$ regional meeting in Los Angeles, in November 1995. One of us (E. G.) has lectured on this material at the International Workshop on Dynamical Systems, held at the Stefan Banach Institute of the Polish Academy of Sciences, in Warsaw, in July 1995.

2. We have circulated a prelimary version of this note, by e-mail, in February 1996. We thank H. Masur for pointing out an error in that version.

\section{References}

[Brs60] L. Bers, Holomorphic differentials as functions of moduli, Bull. Amer. Math. Soc. 66 (1960), 94-97.

[Brm75] J. Birman, Braids, links, and mapping class groups, Ann. Math. Studies 82, Princeton Univ. Press, Princeton, 1975.

[Brd83] A. Beardon, The geometry of discrete groups, Springer-Verlag, New York, 1983.

[Gard87] F. P. Gardiner, Teichmuller theory and quadratic differentials, John Wiley, New York, 1987.

[Gut84] E. Gutkin, Billiard flows on almost integrable polyhedral surfaces, Erg. Theor. Dynam. Sys. 4 (1984), 569-584.

[Gut86] _ Billiards in polygons, Physica 19 D (1986), 311-333.

[Gut95] — Billiards in polygons: survey of recent results, J. Stat. Phys. 83 (1996), 7-26. 
[GH95] E. Gutkin and N. Haydn, Topological entropy of generalized polygon exchanges, Bull. Amer. Math. Soc. 32 (1995), 50-57.

[GH96] _ Topological entropy of polygon exchange transformations and polygonal billiards, Erg. Theor. Dynam. Sys. 16 (1996), in press.

[GJ96] E. Gutkin and C. Judge, Coverings of translation surfaces, prime geodesic theorems, and spectral arithmeticity, preprint, Stanford University (1996).

[GT95] E. Gutkin and S. Troubetzkoy, Directional flows and periodic orbits for polygonal billiards, preprint, USC (1995).

[Jdg95a] C. Judge, On the existence of Maass cusp forms on hyperbolic surfaces with cone points, J. Amer. Math. Soc. 8 (1995), 715-759.

[Jdg95b] _ On the angular moduli of constant curvature surfaces with conical singularities, preprint (1995).

[Kat86] A. Katok, Ergodicity of generic irrational billiards, p. 13, in "Abstracts of workshop on two-manifolds and geometry", MSRI Publ. vol. 1, 1986.

[Kat87] _ , The growth rate for the number of singular and periodic orbits for a polygonal billiard, Comm. Math. Phys. 111 (1987), 151-160.

[KZ75] A. Katok and A. Zemlyakov, Topological transitivity of billiards in polygons, Math. Notes 18 (1975), 760-764.

[KMS] S. Kerckhoff, H. Masur, and J. Smillie, Ergodicity of billiard flows and quadratic differentials, Ann. of Math. 124 (1986), 293-311.

[Krb78] R. Kirby, Problems in low dimensional manifold theory, in Proc. Symp. Pure Math. 32 (1978), 273-312.

[Kra81] I. Kra, On the Nielson-Thurston-Bers type of some self-maps of Riemann surfaces, Acta Math. 146 (1981), 231-270.

[Mas82] H. Masur, Interval exchange transformations and measured foliations, Ann. of Math. 115 (1982), 168-200.

[Mas88] _ _ Lower bounds for the number of saddle connections, and closed trajectories of a quadratic differential, 215-228, in "Holomorphic Functions and Moduli I", MSRI Publ. Vol. 10, Springer-Verlag, New York, 1988.

[Mas90] _ , The growth rate for trajectories of a quadratic differential, Ergod. Theor. Dyn. Sys. 10 (1990), 151-176.

[Nag] S. Nag, Complex analytic theory of Teichmuller spaces, John Wiley, New York, 1988.

[Srk86] P. Sarnak, On cusp forms, Contemp. Math., vol. 53, A.M.S., Providence, R.I. (1986), 393-407.

[Slb54] A. Selberg, Göttingen lectures, 1954.

[Tak77] K. Takeuchi, Arithmetic triangular groups, J. Math. Soc. Japan 29 (1977), 91-106.

[Trs] A. Terras, Harmonic analysis on symmetric spaces, Springer-Verlag, New York, 1985.

[Thurst78] W. Thurston, The geometry and topology of 3-manifolds, Lecture Notes, Princeton University, 1978.

[Thurst88] _ _ On the geometry and dynamics of diffeomorphisms of surfaces, Bull. Amer. Math. Soc. 19 (1988), 417-431.

[Vch82] W. A. Veech, Gauss measures for transformations on the space of interval exchange maps, Ann. of Math. 115 (1982), 201-242.

[Vch89] _ Teichmuller curves in modular space, Eisenstein series, and an application to triangular billiards, Invent. Math. 7 (1989), 553-583.

[Vch92] _ The billiard in a regular polygon, Geom. and Func. Anal. 2 (1992), $341-379$. 
[Vch93] _ _ Flat surfaces, Amer. J. Math. 15 (1993), 589-689.

[Vnk] A. B. Venkov, The spectral theory of automorphic functions, Kluwer, Amsterdam, 1990.

[Vrb96] V. B. Vorobets, Flat structures and billiards in rational polygons, Uspekki Mat. Nauk 51 (1996), 145-146 (in Russian).

[Wrd96] C. Ward, Fuchsian groups and polygonal billiards, Ph. D. Thesis, Rice University (1996).

University of Southern California, Los Angeles, CA 90089-1113

E-mail address: egutkin@math.usc.edu

INDIANA UNIVERSITy, BLOOMINGTON, IN 47405-5701

Current address: Stanford University, Stanford, CA 94305

E-mail address: cjudge@math.stanford.edu 